\title{
GRUPOS DE PERMUTAÇÕES E DE SIMETRIA
}

José Paulo Rodrigues da Silveira ${ }^{1}$, Fernando Pereira Souza

Universidade Federal de Mato Grosso do Sul - Campus de Três Lagoas. ${ }^{1}$ Bolsista do Grupo PET Conexões de Saberes Matemática/CPTL/UFMS. E-mail: josepapt@hotmail.com

\section{RESUMO}

O trabalho apresenta resultados de um estudo sobre "A Teoria dos Grupos" que foi desenvolvido como parte das atividades de pesquisa e apresentações de seminários, vinculado às disciplinas de Álgebra, com o objetivo de entender o conceito de Grupos. Foram estudados alguns conceitos de Relações, Aplicações e Operações importantes para o desenvolvimento do trabalho. Em seguida, foram estudados diversos tipos importantes de grupo, cujo os de maior foco foram os "Grupo de Permutações" e os "Grupos de Simetria do Triângulo Equilátero e do Quadrado".

Palavras-chave: Grupos, Permutações, Simetria, Triângulo Equilátero, Quadrado.

\section{INTRODUÇÃO}

O trabalho apresenta resultados de um estudo sobre o tema "A Teoria dos Grupos", tema de grande importância num curso de Matemática - Licenciatura. Este trabalho foi desenvolvido como parte das atividades de pesquisa do grupo Pet Conexões de Saberes da Universidade Federal de Mato Grosso do Sul no Campus de Três Lagoas. O tema busca desenvolver conteúdos de Matemática do Ensino Básico e da Graduação, complementados por aplicações e exemplos motivadores e está vinculado às disciplinas de Álgebra. O objetivo do presente trabalho é entender os conceitos de Grupos, e explorar os casos particulares dos "Grupos de Permutações" e "Grupos de Simetria do Triângulo Equilátero e do Quadrado".

Para desenvolver o presente trabalho houve a necessidade de desenvolver um trabalho teórico, fazendo uma revisão de vários conceitos de Álgebra, como por exemplo, relações e aplicações, tais como aplicação idêntica e composição de aplicações. Em seguida, foram estudados com bastante atenção alguns conceitos de Operações, necessários ao desenvolvimento do trabalho. Estes conceitos foram estudados com base em Hygino H.Domingues, Gelson lezzi (2003), cujos textos foram utilizados para apresentar, a seguir, os principais conceitos e definições de maneira resumida.

\section{METODOLOGIA}

O trabalho é resultado de uma pesquisa teórica, desenvolvido através de discussões do tema com o orientador e apresentações de seminários como parte das atividades do programa 
PET Conexões de Saberes Matemática - UFMS/CPTL no estudo da Teoria Dos Grupos. O trabalho incluiu uma etapa de leitura e resoluções de exercícios, desenvolvimento das atividades propostas e a tabulação dos resultados obtidos. O estudo e as atividades desenvolvidas foram avaliados através da apresentação de seminários de discussão.

\section{RESULTADOS}

Definição 1: Um sistema matemático constituído de um conjunto não vazio $G$ e uma operação $(x, y) \mapsto x * y$ sobre $\mathrm{G}$ é chamado grupo se essa operação se sujeita aos seguintes axiomas:

\section{- Associatividade}

$(a * b) * c=a *(b * c)$, quaisquer que sejam $a, b, c \in G$

- Existência de Elemento Neutro

Existe um $e \in G$ tal que $a * e=e * a=a$, qualquer que seja $a \in G$;

\section{- Existência de Simétricos}

Para todo $a \in G$ existe um elemento $a^{\prime} \in G$ tal que $a * a^{\prime}=a^{\prime} * a=e$.

Se além disso, ainda se cumprir o axioma da

\section{- Comutatividade}

$a * b=b * a$, quaisquer que sejam $a, b \in G$,

o grupo recebe o nome de grupo comutativo ou abeliano.

Temos que o Grupo aditivo dos inteiros $(\square,+)$ é comutativo, pois $\forall a, b \in \square$, temos que $a+b=b+a \in \square$.

Ainda, o grupo linear de grau $n\left(\mathbb{M}_{n}(\mathbb{K}), \cdot\right)$, não é comutativo se $n>1$, pois, por exemplo, se $A=\left[\begin{array}{cccc}1 & 1 & \ldots & 1 \\ 0 & 1 & \ldots & 1 \\ \vdots & \vdots & & \vdots \\ 0 & 0 & \ldots & 1\end{array}\right]$ e $B=\left[\begin{array}{cccc}1 & 1 & \ldots & 1 \\ 0 & 1 & \ldots & 1 \\ \vdots & \vdots & & \vdots \\ 0 & 0 & \ldots & 1\end{array}\right]$. Então $A B=\left[\begin{array}{ccc}n & \ldots & 1 \\ \vdots & & \vdots \\ 1 & \ldots & 1\end{array}\right] \quad B A=\left[\begin{array}{ccc}1 & \ldots & 1 \\ \vdots & & \vdots \\ 1 & \ldots & n\end{array}\right]$

\section{Grupos de Permutações}


Permutação é um termo utilizado na Teoria dos Grupos para designar uma bijeção de um conjunto nele mesmo. Se E indica um conjunto não vazio, denotaremos por $S(E)$ o conjunto das permutações dos elementos de $\mathrm{E}$.

A composição de aplicações é uma operação sobre $S(E)$, pois se f e g são permutações de $E$, ou seja, se $f: E \rightarrow E$ e $g: E \rightarrow E$ são bijeções, então $g \circ f: E \rightarrow E$ também é uma bijeção. Vejamos a demonstração de tal fato:

DEM: Sejam $x_{1}, x_{2} \in E$ tais que $(g \circ f)\left(x_{1}\right)=(g \circ f)\left(x_{2}\right)$. Então $g\left(f\left(x_{1}\right)\right)=g\left(f\left(x_{2}\right)\right)$ e, como $g$ é injetora, $f\left(x_{1}\right)=f\left(x_{2}\right)$, e do fato de $f$ ser injetora, temos $x_{1}=x_{2}$. Logo, $g \circ f$ é injetora.

Agora, seja $z \in E$. Como $g$ é sobrejetora, $\exists y \in E$ tal que $g(y)=z$. Sendo $f$ sobrejetora, $\exists x \in E$ tal que $f(x)=y$. Assim, temos:

$$
z=g(y)=g(f(x))=(g \circ f)(x) .
$$

Ainda, temos que vale a associatividade para essa operação e que $i_{E}: E \rightarrow E$ (aplicação idêntica de $E)$, que também é uma bijeção, é elemento neutro, pois: $\left(i_{E} \circ f\right)(x)=i_{E}(f(x))=f(x)$, $\forall x \in E$, o que garante que $i_{E} \circ f=f$. Analogamente, temos que $f \circ i_{E}=f$.

Ainda, temos que, pelas definições de aplicações, se $f$ é uma permutação de $E$, então o mesmo ocorre com $f^{-1}$ (aplicação inversa de f). Assim, $f^{-1} \circ f=f \circ f^{-1}=i_{E}$.

Ainda, a ordem de um grupo $(G, *)$, onde $\mathrm{G}$ é finito, é o número de elementos do conjunto G. Caso $\mathrm{G}$ seja um conjunto infinito, dizemos que $(G, *)$ tem ordem infinita.

Desta maneira, temos que $(S(E), \circ)$ é um grupo, chamado de grupo das permutações sobre E. Ainda, esse grupo é Abeliano se, e somente se, sua ordem é 1 ou 2.

De fato, se a ordem é $1, S(E)$ só possui a aplicação idêntica como elemento que, naturalmente, comuta consigo mesma. Se a ordem é 2 e os elementos de $E$ forem $a$ e $b$, então $S(E)$ também só tem dois elementos: a aplicação idêntica e a aplicação que leva $a$ em $b$, e viceversa. Como obviamente esta última aplicação comuta consigo mesma e com $i_{E}$, então $(S(E), \circ)$ também é comutativo neste caso.

Suponhamos agora que a ordem de $S(E)$ é maior que 2 e que, portanto, $E$ tenha mais do que 2 elementos. Designando por $a, b, c$ três elementos distintos de $E$, consideremos as permutações $f$ e $g$ de $S(E)$ definidas da seguinte maneira: 


$$
\begin{aligned}
& f(a)=b, f(b)=a \text { e } f(x)=x, \forall x \neq a, b, \\
& g(a)=c, g(c)=a \text { e } g(x)=x, \forall x \neq a, c .
\end{aligned}
$$

É claro que $f$ e $g$ são permutações de E, pela maneira como foram construídas. Além disso,

$$
\begin{aligned}
& (f \circ g)(a)=f(g(a))=f(c)=c, \\
& (g \circ f)(a)=g(f(a))=g(b)=b .
\end{aligned}
$$

Isso mostra que $f \circ g \neq g \circ f$ e, portanto, que $S(E)$ não é comutativo.

\section{Grupo Simétrico de Grau n}

O Grupo em que $E=\{1,2, \ldots, n\}$, com $n \geq 1$ é um caso particular do grupo de permutações. Neste caso, costuma-se utilizar a notação $S_{n}$ para indicar o conjunto das permutações sobre E. Tal grupo, $\left(S_{n}, \circ\right)$ denomina-se Grupo Simétrico de Grau n.

Pela análise combinatória, podemos concluir que esse grupo tem ordem $n$ !.

Notação: Seja $g \in S_{n}$ e $g(1)=i_{1}, g(2)=i_{2}, \ldots, g(n)=i_{n}$ então usa-se a notação:

$$
g=\left[\begin{array}{cccc}
1 & 2 & \ldots & n \\
i_{1} & i_{2} & \ldots & i_{n}
\end{array}\right]
$$

Vejamos o grupo $S_{3}$. Façamos

$$
\begin{gathered}
S_{3}=\left\{f_{0}=\left(\begin{array}{lll}
1 & 2 & 3 \\
1 & 2 & 3
\end{array}\right), f_{1}=\left(\begin{array}{lll}
1 & 2 & 3 \\
2 & 3 & 1
\end{array}\right), f_{2}=\left(\begin{array}{lll}
1 & 2 & 3 \\
3 & 1 & 2
\end{array}\right),\right. \\
\left.g_{1}=\left(\begin{array}{lll}
1 & 2 & 3 \\
1 & 3 & 2
\end{array}\right), g_{2}=\left(\begin{array}{lll}
1 & 2 & 3 \\
3 & 2 & 1
\end{array}\right), g_{3}=\left(\begin{array}{lll}
1 & 2 & 3 \\
2 & 1 & 3
\end{array}\right)\right\} .
\end{gathered}
$$

Observemos como se obtém $f_{1} \circ g_{3}$ por exemplo:

$$
f_{1} \circ g_{3}=\left(\begin{array}{lll}
1 & 2 & 3 \\
2 & 3 & 1
\end{array}\right) \circ\left(\begin{array}{lll}
1 & 2 & 3 \\
2 & 1 & 3
\end{array}\right)=\left(\begin{array}{lll}
1 & 2 & 3 \\
3 & 2 & 1
\end{array}\right)=g_{2} .
$$

De modo análogo, obtemos as demais composições. Feito isso, observemos a tábua deste grupo: 


\begin{tabular}{|l|llllll|}
\hline$\circ$ & $f_{0}$ & $f_{1}$ & $f_{2}$ & $g_{1}$ & $g_{2}$ & $g_{3}$ \\
\hline$f_{0}$ & $f_{0}$ & $f_{1}$ & $f_{2}$ & $g_{1}$ & $g_{2}$ & $g_{3}$ \\
$f_{1}$ & $f_{1}$ & $f_{2}$ & $f_{0}$ & $g_{3}$ & $g_{1}$ & $g_{2}$ \\
$f_{2}$ & $f_{2}$ & $f_{0}$ & $f_{1}$ & $g_{2}$ & $g_{3}$ & $g_{1}$ \\
$g_{1}$ & $g_{1}$ & $g_{2}$ & $g_{3}$ & $f_{0}$ & $f_{1}$ & $f_{2}$ \\
$g_{2}$ & $g_{2}$ & $g_{3}$ & $g_{1}$ & $f_{2}$ & $f_{0}$ & $f_{1}$ \\
$g_{3}$ & $g_{3}$ & $g_{1}$ & $g_{2}$ & $f_{1}$ & $f_{2}$ & $f_{0}$ \\
\hline
\end{tabular}

Temos que este grupo não é abeliano. Basta observar que $f_{1} \circ g_{1} \neq g_{1} \circ f_{1}$. Podemos observar também que o conjunto $C_{3}=\left\{f_{0}, f_{1}, f_{2}\right\}$, também é um grupo quando considerado com a composição de permutações. De fato, além de ser fechado para a operação, vale a associatividade porque vale em $S_{3}$, o elemento neutro é a permutação $f_{0}$, além disso, $f_{0}^{-1}=f_{0}, f_{1}^{-1}=f_{2}, f_{2}^{-1}=f_{1}$.

\section{Grupos de Simetrias}

\section{(i) $\quad$ grupo $S_{\Delta}$ das simetrias espaciais de um triângulo equilátero.}

Considere um triângulo equilátero e denote os vértices por 1,2,3. Seja $G$ o baricentro do triângulo, ou seja, se $x, y, z$ são as retas do espaço passando pelas medianas do triângulo, então $G$ é a intercessão das medianas conforme a figura abaixo:

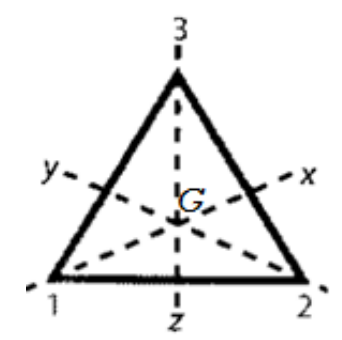

As transformações que preservam o triângulo são:

- $R_{0}, R_{1}, R_{2}$ : As rotações planas centradas em $G$, no sentido anti-horário, de ângulos zero, $\frac{2 \pi}{3}, \frac{4 \pi}{3}$, respectivamente.

- $X, Y, Z$ : As rotações espaciais de ângulo $\pi$ com os eixos $x, y, z$, respectivamente.

Vejamos geometricamente, como se obtém $R_{1} \circ Y$ e $Y \circ R_{1}$, por exemplo: 

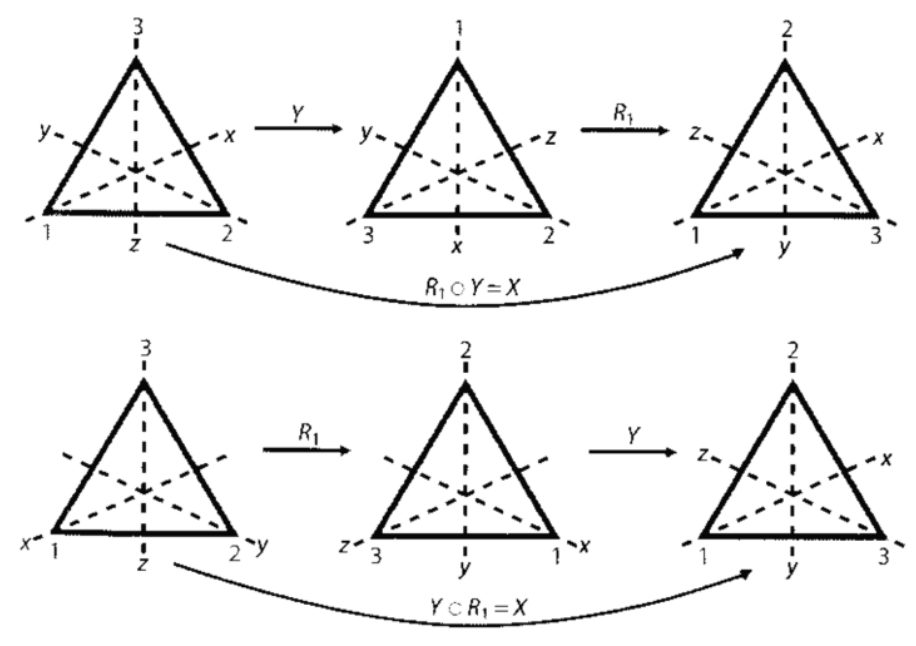

É fácil ver através da tábua que $S_{\Delta}$ é um grupo. Vejamos:

\begin{tabular}{|c|c|c|c|c|c|c|}
\hline$\circ$ & $R_{0}$ & $R_{1}$ & $R_{2}$ & $X$ & $Y$ & $Z$ \\
\hline$R_{0}$ & $R_{0}$ & $R_{1}$ & $R_{2}$ & $X$ & $Y$ & $Z$ \\
\hline$R_{1}$ & $R_{1}$ & $R_{2}$ & $R_{0}$ & $Z$ & $X$ & $Y$ \\
\hline$R_{2}$ & $R_{2}$ & $R_{0}$ & $R_{1}$ & $Y$ & $Z$ & $X$ \\
\hline$X$ & $X$ & $Z$ & $Y$ & $R_{0}$ & $R_{2}$ & $R_{1}$ \\
\hline$Y$ & $Y$ & $X$ & $Z$ & $R_{1}$ & $R_{0}$ & $R_{2}$ \\
\hline$Z$ & $Z$ & $Y$ & $X$ & $R_{2}$ & $R_{1}$ & $R_{0}$ \\
\hline
\end{tabular}

Por meio dela se verifica o fechamento, que $R_{0}$ é elemento neutro e que $R_{0}^{-1}=R_{0}, R_{1}^{-1}=R_{2}, R_{2}^{-1}=R_{1}, X^{-1}=X, Y^{-1}=Y$ e $Z^{-1}=Z$. Valendo a associatividade por se tratar de composição de transformações, logo trata-se de um grupo.

Denotamos esse grupo por $S_{\Delta}=\left\{R_{0}, R_{1}, R_{2}, X, Y, Z\right\}$. Ainda, como a tábua de $S_{\Delta}$ não é simétrica em relação à diagonal principal, então ele não é comutativo.

\section{(ii) $\quad 0$ grupo $D_{\square}$ das simetrias espaciais de um quadrado}

Seja um quadrado com vértices consecutivos 1,2,3,4. Denotemos por $O$ centro de gravidade do quadrado e chame de $x, y, z, w$ as retas do espaço determinadas pelas diagonais e pelas mediatrizes do quadrado 


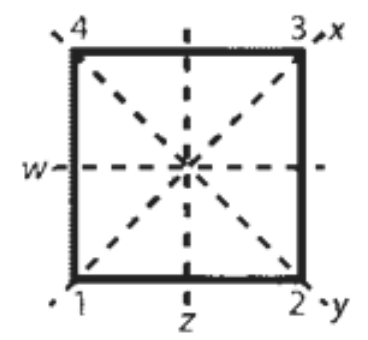

As transformações espaciais que preservam o quadrado são:

- $R_{0}, R_{1}, R_{2}, R_{3}$ : as rotações planas centradas em O, no sentido anti-horário, de ângulos zero, $\frac{\pi}{2}, \pi, \frac{3 \pi}{2}$, respectivamente.

- $X, Y, Z, W$ : as rotações espaciais de ângulo $\pi$ com os eixos $x, y, z, w$, respectivamente.

Vejamos, geometricamente, como se obtém $Z \circ R_{2}$ e $R_{2} \circ Z$ :
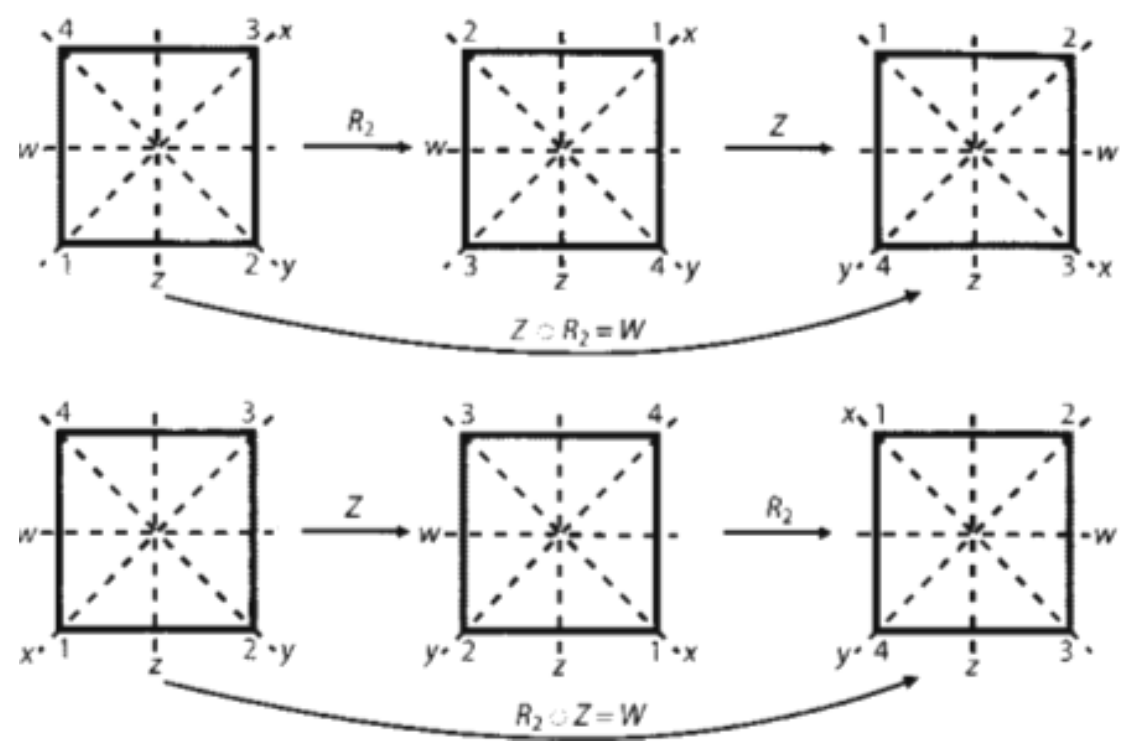

Efetuando-se as demais composições, a tabela obtida é a seguinte:

\begin{tabular}{|c|c|c|c|c|c|c|c|c|}
\hline$\circ$ & $R_{0}$ & $R_{1}$ & $R_{2}$ & $R_{3}$ & $X$ & $Y$ & $Z$ & $W$ \\
\hline$R_{0}$ & $R_{0}$ & $R_{1}$ & $R_{2}$ & $R_{3}$ & $X$ & $Y$ & $Z$ & $W$ \\
\hline$R_{1}$ & $R_{1}$ & $R_{2}$ & $R_{3}$ & $R_{0}$ & $Z$ & $W$ & $Y$ & $X$ \\
\hline$R_{2}$ & $R_{2}$ & $R_{3}$ & $R_{0}$ & $R_{1}$ & $Y$ & $X$ & $W$ & $Z$ \\
\hline$R_{3}$ & $R_{3}$ & $R_{0}$ & $R_{1}$ & $R_{2}$ & $W$ & $Z$ & $X$ & $Y$ \\
\hline$X$ & $X$ & $Z$ & $Y$ & $W$ & $R_{0}$ & $R_{2}$ & $R_{1}$ & $R_{3}$ \\
\hline$Y$ & $Y$ & $W$ & $X$ & $Z$ & $R_{2}$ & $R_{0}$ & $R_{3}$ & $R_{1}$ \\
\hline$Z$ & $Z$ & $Y$ & $Z$ & $X$ & $R_{3}$ & $R_{1}$ & $R_{0}$ & $R_{2}$ \\
\hline$W$ & $W$ & $X$ & $Z$ & $Y$ & $R_{1}$ & $R_{3}$ & $R_{2}$ & $R_{0}$ \\
\hline
\end{tabular}


Através desta tábua temos que a composição de simetrias é uma operação em $D_{\square}$. A associatividade da operação vale por se tratar de particular composição de aplicação, temos $R_{0}$ como elemento neutro e todos os elementos possuem simétrico. Logo, $\left(D_{\square}, \circ\right)$ é um grupo. Este grupo não é abeliano, pois, por exemplo, $X \circ Z=R_{1}$ e $Z \circ X=R_{3}$.

\section{DISCUSSÃO}

O conceito de simetria de um triângulo e de um quadrado pode ser estendido naturalmente para um polígono regular qualquer de $n$ lados. Temos que o número das simetrias de um polígono regular de $\mathrm{n}$ lados é o dobro do número de lados, portanto $2 n$ no caso geral.

Para descrever essas simetrias, denotemos os vértices do polígono consecutivamente por $1,2, \ldots, n$ e o conjunto das simetrias por $D_{n}$. Duas simetrias bastam para geral $D_{n}$ : a rotação $R$ de $\frac{2 \pi}{\mathrm{n}}$ radianos em torno do centro $O$ do polígono
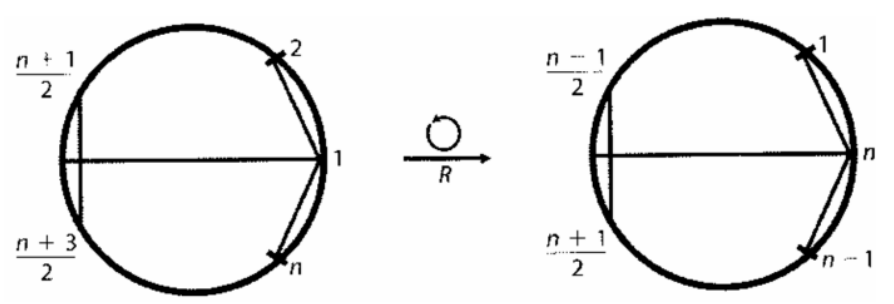

e a reflexão $X$ de $\pi$ radianos em torno de $x$ pelo vértice 1 e pelo centro do polígono (Consideremos $\mathrm{n}$ ímpar)
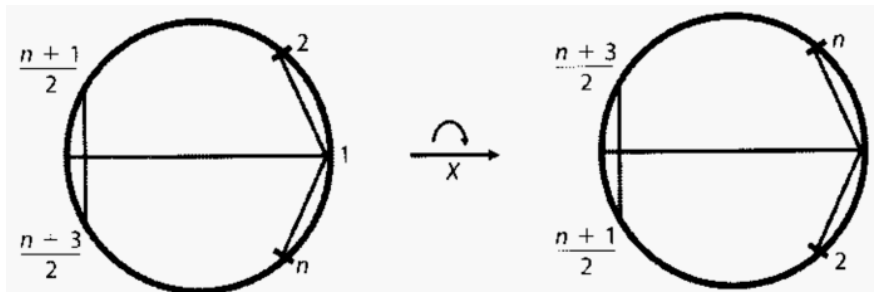

Isto posto, pode-se demonstrar que o conjunto das simetrias do polígono é

$$
D_{n}=\left\{R^{0}, R, R^{2}, \ldots, R^{n-1}, X, X \circ R, X \circ R^{2}, \ldots, X \circ R^{n-1}\right\}
$$

\section{CONCLUSÃO}

Através do trabalho foram obtidos resultados que permitem um aprofundamento em estudos sobre a 'Teoria dos Grupos', bem como uma iniciação em conteúdos básicos de Álgebra Linear, possibilitando assim um maior conhecimento sobre áreas diversas de matemática. 
Encontro de Ensino, Pesquisa e Extensão, Presidente Prudente, 21 a 24 de outubro, 2013

\section{REFERÊNCIAS}

DOMINGUES, Hygino H; IEZZI, Gelson. Álgebra Moderna- 4. Edição reformulada - São Paulo: Atual, 2003.

GARCIA, Arnaldo e LEQUAIN, Yves. Elementos de Álgebra. Rio de Janeiro: Projeto. Euclides, IMPA, 2005.

GONÇALVES, Adilson, Introdução à Álgebra, 5.ed, IMPA, 2011. 